\title{
Development of a Method Using Image Analysis for the Measurement of Cellulose-Binding Domains Adsorbed onto Cellulose Fibers
}

\author{
Ricardo Pinto, ${ }^{\dagger}$ António L. Amaral, ${ }^{\dagger, \dagger}$ Joana Carvalho, ${ }^{\dagger}$ Eugénio C. Ferreira, ${ }^{\dagger}$ Manuel Mota, ${ }^{\dagger}$ and \\ Miguel Gama*,†
}

IBB-Institute for Biotechnology and Bioengineering, Centre of Biological Engineering, Universidade do Minho, Campus de Gualtar 4710-057, Braga, Portugal

\begin{abstract}
The surface concentration of CBD-FITC conjugates, adsorbed on cellulose fibers, was determined by image analysis. The program consists of two scripts, the first dedicated to the elaboration of the calibration curve. The emission of fluorescent light, detected by image analysis, is correlated with the concentration of CBD solutions. This calibration is then used (second script) to determine the concentration of CBDs adsorbed on cellulosic fibers. This method allows the direct estimation of the surface concentration of adsorbed CBDs, which usually is not accurately calculated from depletion studies, since the surface area is hardly known. By observing different spots in the surface of the fibers, site-specific information is obtained. It was verified that the physically heterogeneous fibers exhibit different amounts of adsorbed CBDs.
\end{abstract}

\section{Introduction}

The majority of cellulases have a modular assembly, formed by a catalytic domain, a cellulose-binding domain (CBD), and a highly glycosilated linker (1). CBDs have the ability of enhancing the enzyme catalytic activity, by increasing its concentration on the insoluble cellulose surface (2-4). The CBD from Cellobiohydrolase I (Trichoderma reesei) has an amino acid sequence that includes three aromatic residues (tyrosine) that seem to be implicated in the adsorption to cellulose (5). Carrard et al. (6) showed that different CBDs adsorb to different sites on the surface of crystalline cellulose. The direct measurement of CBDs adsorbed on fibers with different properties, such as crystallinity or surface area, would therefore be very useful in the characterization of biochemical properties of CBDs.

Pala et al. (7) showed that the presence of CBD, during paper production, modified the technological properties of the pulp and paper. In the present work, having in mind its biotechnological relevance, we aim at quantifying the CBD adsorbed on cellulosic surfaces, with the help of the fluorescent complex CBD-FITC. The covalent labeling of the linker terminal amine of the carbohydrate-binding module (CBM) with FITC was demonstrated not to affect its cellulose binding ability $(8)$. The use of image analysis methodologies was then validated as a means to quantify the adsorbed CBD-FITC.

Image processing and analysis techniques have been shown to be an extremely useful tool in a vast number of applications, because they allow the elimination of human analysis subjectivity and also the extraction of quantitative data that would be very difficult or even impossible to acquire by other means. As a matter of fact, there is a stepwise increment in the use of image analysis methodologies in processes that involve identification or even fluorescence quantification, in both microorganisms and fluorescent probes $(9,10)$.

* To whom correspondence should be addressed. Fax: +351253678986. E-mail: fmgama@deb.uminho.pt.

$\dagger$ Institute for Biotechnology and Bioengineering.

$\doteqdot$ Departamento de Tecnologia Química, ESTIG, IPB, Apartado 1038, 5301-854 Bragança, Portugal.

\section{Materials and Methods}

2.1. Chemicals. Fluorescein isothiocyanate (FITC) was obtained from Sigma, and cellulosic fibers CF11 from Whatman (pure cellulose). All chemicals were of the highest purity available.

2.2. CBD Production. The CBDs were prepared according to the following methodology. The Celluclast commercial enzymatic preparation (Novozymes A/S, Denmark) was digested with papain (1:1200, on a protein basis). The resulting CBDs were separated by ultrafiltration through a $10 \mathrm{kDa}$ membrane (Pellicon 2 TFF System from Millipore, USA) and concentrated by precipitation with ammonium sulfate (Merck, Darmstadt, Germany). After dialysis, CBDs were further purified on a Sepharose Fast-Flow gel (Amersham Pharmacia Biotech AB, Sweden); the non-adsorbed protein (CBD) was collected and lyophilized. Although produced by proteolysis, the obtained CBD is rather pure (11). It is the CBD from $T$. reesei $\mathrm{CBHI}$, with a heavily glycosilated linker attached. The purity of this protein has been demonstrated by $\mathrm{N}$-terminal sequencing and MALDI-TOF.

2.3. CBD-FITC Production. The conjugation of CBD with the labeling probe was carried out by mixing $20 \mu \mathrm{g}$ of FITC per $\mathrm{mg}$ of CBD (in a concentration of $2 \mathrm{mg}_{\text {protein }} \cdot \mathrm{mL}^{-1}$ in 0.1 M HEPES buffer, $\mathrm{pH}$ 9.0). This solution was incubated overnight in the dark, at room temperature, with magnetic stirring. To eliminate the unbound FITC, the labeled CBD mixture was filtered through a column packed with BIO-GEL P-4 (BIO-RAD, Hercules, USA), previously equilibrated with $50 \mathrm{mM}$ sodium acetate buffer (Panreac, Barcelona, Spain).

2.4. CBD Adsorption Isotherm. Suspensions of Whatman CF11 fibers, with a concentration of $10 \mathrm{mg}$ per $\mathrm{mL}$ in sodium acetate buffer (50 mM, pH 5.0), were incubated for $2 \mathrm{~h}$ at 5 ${ }^{\circ} \mathrm{C}$, with magnetic stirring, in the presence of different CBD concentrations $\left(\mathrm{CBD}_{\text {Initial }}, \mu \mathrm{M}\right)$. The fibers were then centrifuged at $4000 \mathrm{rpm}$ for $10 \mathrm{~min}$. The $\mathrm{CBD}$ concentration in the supernatant $\left(\mathrm{CBD}_{\text {Free }}, \mu \mathrm{M}\right)$ was measured in a Jasco FP6200 spectrofluorimeter, operated at an emission and excitation wavelengths of 341 and $275 \mathrm{~nm}$, respectively. The apparatus 
was calibrated using CBD solutions with known concentrations (BCA protein assay, Pierce, using BSA as protein standard). The bound CBD $\left(\mu \mathrm{mol} \cdot \mathrm{g}^{-1}\right)$ was calculated using the following equation:

$$
\mathrm{CBD}_{\text {Bound }}=\frac{\left[\mathrm{CBD}_{\text {Initial }}\right]-\left[\mathrm{CBD}_{\text {Free }}\right]}{m_{\mathrm{CF} 11}} \cdot V_{\mathrm{R}}
$$

where $V_{\mathrm{R}}(\mathrm{L})$ corresponds to the volume of buffer used and $m_{\mathrm{CF} 11}(\mathrm{~g})$ is the fibers mass.

The values of the adsorbed versus free CBDs were correlated, by nonlinear regression analysis, with the Langmuir adsorption isotherm (12):

$$
\mathrm{CBD}_{\text {Bound }}=\frac{\mathrm{CBD}_{\mathrm{Max}} \cdot k_{\mathrm{a}} \cdot \mathrm{CBD}_{\text {Free }}}{1+k_{\mathrm{a}} \cdot \mathrm{CBD}_{\text {Free }}}
$$

where $\mathrm{CBD}_{\text {Max }}$ is the maximum molar amount of adsorbed protein $\left(\mu \mathrm{mol} \cdot \mathrm{g}^{-1}\right)$ and $k_{\mathrm{a}}$ is the adsorption equilibrium constant $\left(\mu \mathrm{M}^{-1}\right)$.

2.5. Production of Cellulose Films and CBD Adsorption. Cellulose films were obtained by evaporation, on a glass surface, of a solution of cellulose acetate in acetone. Then the films were saponified in $0.1 \mathrm{M}$ ethanolic solution of sodium hydroxide at $40{ }^{\circ} \mathrm{C}$ for $2 \mathrm{~h}$. After saponification, the films were rinsed in ultrapure water.

AFM topography images $(512 \times 512$ pixels $)$ of a cellulose film were obtained in tapping mode using a MultiMode SPM (Digital Instruments) apparatus.

Adsorption assays of FITC-labeled CBD were carried out at $4{ }^{\circ} \mathrm{C}$. The conjugates $\left(400 \mu \mathrm{g} \cdot \mathrm{mL}^{-1}\right)$ were allowed to adsorb on the cellulose film (circles with $\phi 2 \mathrm{~mm}$ ), in $50 \mathrm{mM}$ sodium acetate buffer (final volume of $4 \mathrm{~mL}$ ), with continuous magnetic stirring in the dark for $2 \mathrm{~h}$. The cellulose films with bound CBD were then washed with sodium acetate buffer to remove the non-adsorbed CBD-FITC.

Adsorption on a single side of the cellulose films was achieved by placing a drop of the CBD-FITC solution on the surface of the film. After $30 \mathrm{~min}$, the film was washed with sodium acetate buffer, and fluorescent images were taken from both sides using an Axioskop microscope (Zeiss, Oberkochen, Germany) with an AxioCam HRc attached camera (Zeiss, Oberkochen, Germany). This experiment was carried out to compare the fluorescent intensity observed, depending on whether the radiation has to cross (or not) the cellulose film (according to the side of the film, with and without CBDs, exposed to the camera).

2.6. Image Analysis: Calibration. Fluorescence microscopy observations were performed in an Axioskop microscope (objective with $40 \mathrm{X}$ magnification and numerical aperture of 0.75), with an AxioCam HRc attached camera. All images were acquired at $1300 \times 1030$ pixels and 24 bits color depth $(8$ bits per channel) by the AxioVision 3.1 software (Zeiss, Oberkochen, Germany). Solutions with different CBD-FITC concentrations, determined using the BCA protein assay kit (Pierce), were used to perform calibrations in an Improved Neubauer chamber. Images obtained with these solutions were related to the quantity of $\mathrm{CBD}\left(\mathrm{Q}_{\mathrm{CBD}}, \mathrm{mol} \cdot \mathrm{mm}^{-2}\right)$ by the following equation:

$$
\mathrm{Q}_{\mathrm{CBD}}=\frac{[\mathrm{CBD}] \cdot 0.10}{8.43 \times 10^{9}}
$$

where $[\mathrm{CBD}]$ is the concentration of CBD-FITC $\left(\mathrm{mg} \cdot \mathrm{mL}^{-1}\right)$, 0.10 is the depth of the liquid in the Neubauer chamber $(\mathrm{mm})$, and $8.43 \times 10^{9}$ is the molecular weight of the CBD used $\left(\mu \mathrm{g} \cdot \mathrm{mol}^{-1}\right)$ as determined by MALDI (11).

To estimate the quantity of CBD-FITC adsorbed to cellulose fibers, an image processing and analysis program was developed in Matlab (The Mathworks, Inc, Natick). This program consists of two scripts, the first focused on the calibration curve, and the second on the quantification of the adsorbed CBD-FITC.

To perform the calibration, 5-10 different concentrations of CBD and different acquisition times (in the range of 200-3000 $\mathrm{ms}$ ) were used in order to determine the adsorbed CBDs in a range of concentration as large as possible. The first step of calibration consisted in the determination of the RGB channels merging function that maximizes the sensitivity to small fluorescence variations. This merging function $\left(I=f_{\mathrm{R}} I_{\mathrm{R}}+f_{\mathrm{G}} I_{\mathrm{G}}\right.$ $\left.+f_{\mathrm{B}} I_{\mathrm{B}}\right)$ allows the fusion of the three channels images $\left(I_{\mathrm{R}}, I_{\mathrm{G}}\right.$, and $I_{\mathrm{B}}$ ) in one single image of intensity $I$, taking in account the sensitivity factors for each channel $\left(f_{\mathrm{R}}, f_{\mathrm{G}}\right.$, and $\left.f_{\mathrm{B}}\right)$, calculated as defined ahead. The average intensity was determined for each RGB channel, for each different CBD-FITC concentration and capture time. The channel's sensitivity factor was calculated as the ratio $f_{\mathrm{R}}=I_{\mathrm{R}} /\left(I_{\mathrm{R}}+I_{\mathrm{G}}+I_{\mathrm{B}}\right), f_{\mathrm{G}}=I_{\mathrm{G}} /\left(I_{\mathrm{R}}+I_{\mathrm{G}}+I_{\mathrm{B}}\right)$, and $f_{\mathrm{B}}$ $=I_{\mathrm{B}} /\left(I_{\mathrm{R}}+I_{\mathrm{G}}+I_{\mathrm{B}}\right)$, for each image capture time and CBDFITC concentration. Finally, the general sensitivity factors were determined, for each channel, by averaging the asymptotic values for all of the capture times and for each concentration. By applying these factors to each channel of the acquired images, a unique single-channel (gray scale) image of maximum sensitivity was obtained.

The ultimate objective of the first Matlab script consisted of the elaboration of a calibration curve for each data acquisition time, correlating the CBD-FITC concentration to the intensity value and spatial localization in the image (due to the nonuniform illumination of the field). Therefore, for each acquired image, corresponding to a specific CBD-FITC concentration and data acquisition time, the three color channels are merged into one single image, by the aforementioned merging function. Because of execution time and virtual memory limitations, it was necessary to resize each image to $1 / 5$ of its original size (from $1300 \times 1030$ pixels to $260 \times 206$ pixels). Since the original images are relatively soft at the transition level between neighbor pixels, the image reduction does not imply significant errors.

The FITC fluorophore is known to photobleach due to several factors, such as concentration, exposure time, or light intensity among others (13). This bleaching effect can be described using the monoexponential equation:

$$
I=I_{0} \cdot \mathrm{e}^{-k_{d} \cdot t}
$$

where $I_{0}$ and $I$ are the fluorescence intensities before and after $t$ seconds of exposure and $k_{\mathrm{d}}$ is the photobleaching constant rate, $\mathrm{s}^{-1}(14,15)$.

The images obtained with the AxioCam sensor correspond to the total fluorescence detected during the exposure time $(t)$ or, graphically, to the gray shaded area in the inset of Figure 1. Thus, integrating eq 4:

$$
I_{\mathrm{A}}=\frac{I_{0}}{k_{\mathrm{d}}} \cdot\left(1-\mathrm{e}^{-k_{d} \cdot t}\right)
$$

The values of $I_{0}$ and $k_{\mathrm{d}}$ parameters are determined from a nonlinear regression of eq 5 (Figure 1 ), where $I_{\mathrm{A}}$ is the fluorescence emission of the calibration images. This regression is made at each pixel position, for the various CBD-FITC concentrations. The values of $I_{0}$ are then multiplied by the 


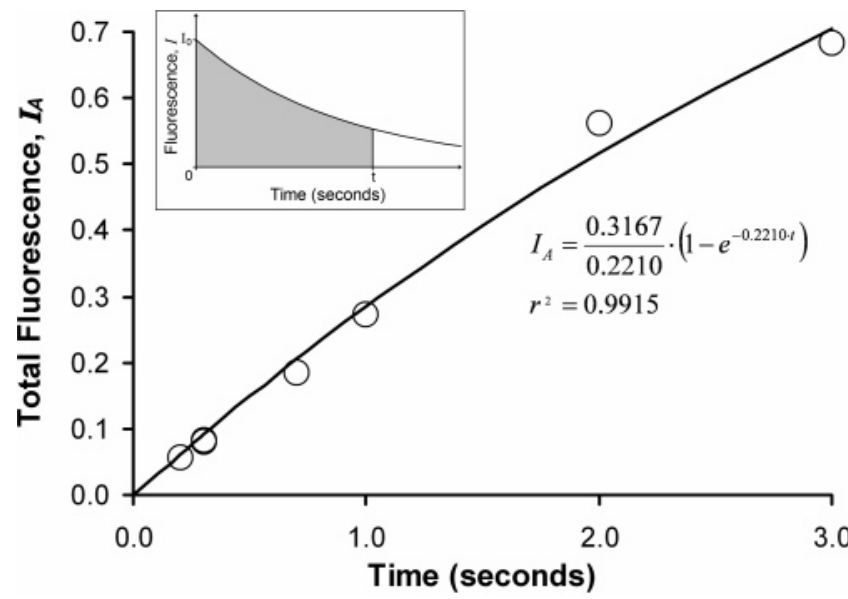

Figure 1. Values of fluorescence intensity $\left(I_{\mathrm{A}}\right)$ versus exposure time, obtained using a CBD - FITC concentration of $10.14 \times 10^{-13} \mathrm{~mol} \cdot \mathrm{mm}^{-2}$ (or $85.5 \mu \mathrm{g} \cdot \mathrm{mL}^{-1}$ ), and the corresponding nonlinear regression fitting. Inset: photobleaching curve of a monoexponential correlation, the model used in the calculation of $I_{\mathrm{A}}$.

exposure time of the images to be quantified, in order to obtain the total unbleached fluorescence (area) for that exposure, and a linear regression is performed for $I_{0} \cdot t$ versus the concentration of CBD-FITC (Figure 2). The coefficient values for each pixel are then saved in a Matlab native format, to be used in the second script.

2.7. Image Analysis: CBD Quantification. The second Matlab script was devoted to the determination of the CBDFITC concentration in cellulose films/fibers. Images were obtained, exhibiting the appropriate level of signal intensity. A convenient capture time was selected, based on the criteria of obtaining the maximum (at non-saturation) signal intensity, thus maximizing the fibers image sensitivity. For each of the acquired images, the three color channels were merged into one single image, which was then resized to $1 / 5$ of its original size (from $1300 \times 1030$ pixels to $260 \times 206$ pixels). Finally, the pixel intensity values of the fibers image were converted to their corresponding concentration values, by the use of the appropriate calibration model. Then, the fiber-target zone(s) were selected for the determination of the adsorbed CBD-FITC average concentration. Since the fiber-target zone(s) have uniform intensities, the size reduction does not introduce significant errors. The average concentrations of the fiber-target zone(s) are then saved in a text file, together with their cardinal positions.

\section{Results and Discussion}

The calibration of the fluorescence obtained in microscopic images has been done by other authors, using polyvinylalcohol films with embedded fluorescein (16). This approach presents some inconveniencies: a shift in the fluorescent emission may occur, due to the different medium surrounding the fluorescent probe (a polymer film in the calibration, water in the case of the studies with CBD-FITC); second, this is a more laborious approach. Indeed, the calibration procedure developed in this work, based on the use of solutions with the fluorescent probe, in an Improved Neubauer chamber, allowed both a higher accuracy and a simpler methodology.

The three channel images $\left(I_{\mathrm{R}}, I_{\mathrm{G}}\right.$, and $\left.I_{\mathrm{B}}\right)$ were merged into a single image of intensity $I$, using sensitivity factors experimentally determined for each channel $\left(f_{\mathrm{R}}, f_{\mathrm{G}}\right.$, and $\left.f_{\mathrm{B}}\right)$, for each CBD-FITC concentration and image capture time. Saturated images (exhibiting intensity points close to 255 in any channel)

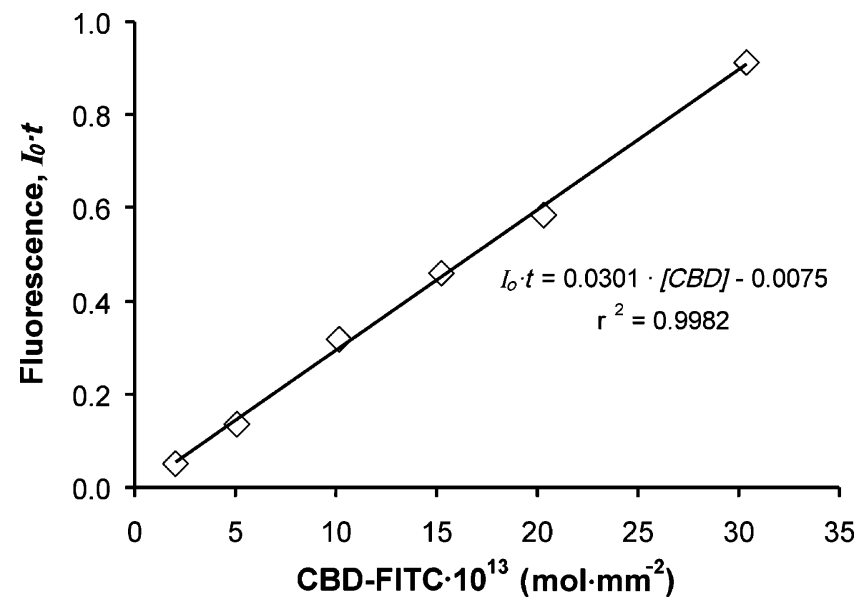

Figure 2. Values of fluorescence intensity as a function of the CBDFITC concentration.

were excluded from the sensitivity factors determination. For instance, at $300 \mathrm{~ms}$ capture time and for concentrations above $100 \times 10^{-13} \mathrm{~mol} \cdot \mathrm{mm}^{-2}$, the green channel becomes saturated (Figure 3).

As described before, the sensitivity factors were calculated from the ratio between each channel intensity and the sum of all channels' intensities. It was verified that, for values below the image saturation threshold (255), the sensitivity factor is a constant value, independent of the CBD-FITC concentration and capture time (Figure 3). The obtained values are presented in Table 1. The sensitivity factors calculated are constant and independent of the capture time. Therefore, the merging function could be determined as:

$$
I=0.208 \times I_{\mathrm{R}}+0.695 \times I_{\mathrm{G}}+0.096 \times I_{\mathrm{B}}
$$

Several analyses were performed using different capture times and CBD-FITC concentrations. Good correlations were obtained using the calculated sensitivity factors. As an example, images obtained for different CBD-FITC concentrations ( $I$ vs $x y$ pixel), using a capture time of $300 \mathrm{~ms}$, are shown in Figure 4. This figure reveals a nonuniform background illumination, resulting in a significant variation of the intensities measured at different pixel locations. Several authors $(14,17,18)$ used a "mask" image to correct this type of nonuniformity. In this work, this approach was not used, since it gives rise to color artifacts in the image borders. Nevertheless, with the strategy used in the calibration, this spatial variation is overcome. Figure 4 also shows the proportional increase of the measured intensity with the CBD-FITC concentration.

To ensure that the color intensity values were not dependent on the quantity or height of CBD solution used on the Neubauer chamber, two additional experiments were performed. In the first one, the CBD-FITC quantification was carried out using volumes of $3.34,6.66$, and $10.00 \mu \mathrm{L}$. The same CBD-FITC solution was used in each case; although the used volume varies, the theorical surface concentration of CBDs is the same, $10 \times$ $10^{-13} \mathrm{~mol} \cdot \mathrm{mm}^{-2}$, since the depth of the solution ( $z$-axis in the Neubauer chamber) is the same. The surface concentration calculated for the three different volumes of protein solution is shown in Figure 5a. This figure shows a small increase in the estimated concentration, toward the expected value of $10 \times$ $10^{-13} \mathrm{~mol} \cdot \mathrm{mm}^{-2}$, raising the volume $3.34 \mu \mathrm{L}$ to $10 \mu \mathrm{L}$. Therefore, and for subsequent analysis, a $10 \mu \mathrm{L}$ volume was chosen in the development of the calibration models, because of the higher accuracy. In the second experiment, the quantification was carried out using CBDs solutions with three different 

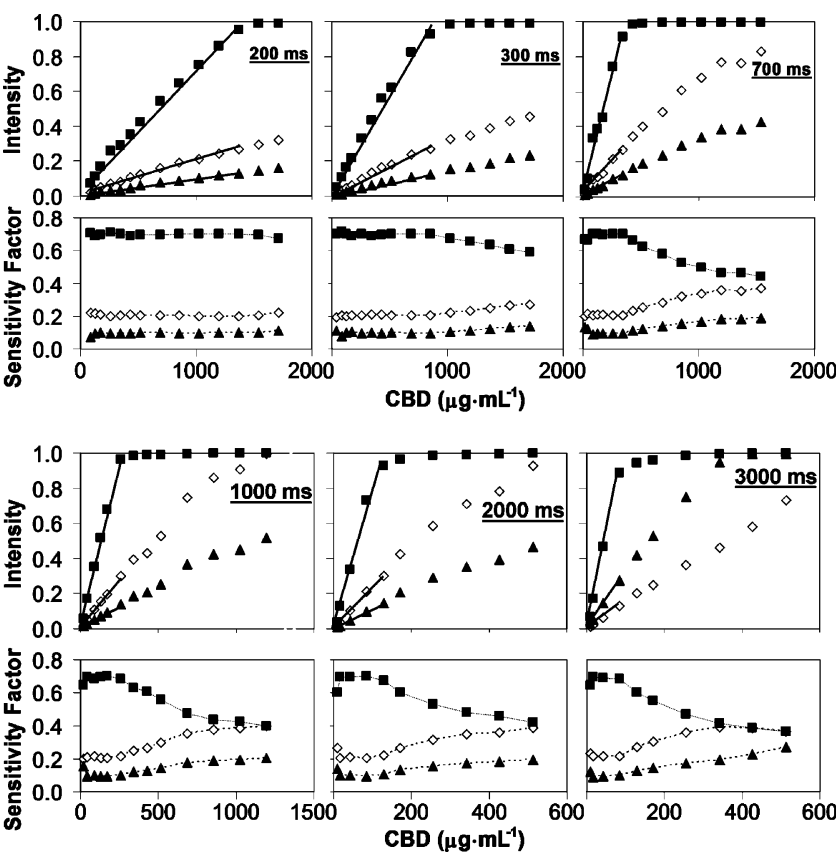

Figure 3. Intensity and sensitivity factors variation with $\mathrm{CBD}$ concentration for the studied capture times, obtained by averaging a $10 \times 10$ pixel area at the center of each original images (continuous lines connect the values used for the calibration model): $(\diamond)$ red channel; ( $\mathbf{\square})$ green channel; ( $\mathbf{\Lambda})$ blue channel.

Table 1. Sensitivity Factor Values for Each Channel as a Function of Capture Time

\begin{tabular}{lccc}
\hline time $(\mathrm{ms})$ & $\mathrm{f}_{\mathrm{R}}$ & $\mathrm{f}_{\mathrm{G}}$ & $\mathrm{f}_{\mathrm{B}}$ \\
\hline 200 & 0.206 & 0.699 & 0.095 \\
300 & 0.204 & 0.700 & 0.097 \\
700 & 0.206 & 0.699 & 0.094 \\
1000 & 0.210 & 0.692 & 0.098 \\
2000 & 0.208 & 0.692 & 0.100 \\
3000 & 0.215 & 0.690 & 0.095 \\
average & 0.208 & 0.695 & 0.096
\end{tabular}

heights (L, 2L, and 3L, obtained using different volumes of liquid in a glass container). As can be observed in Figure 5b, the quantification resulted in proportional values, showing that the camera detects all the fluorescence emitted from the $z$-axis, perpendicular to the observed CBD solution.

To validate further the methodology, measurements of CBDFITC adsorbed to cellulose films were made. The photobleach-

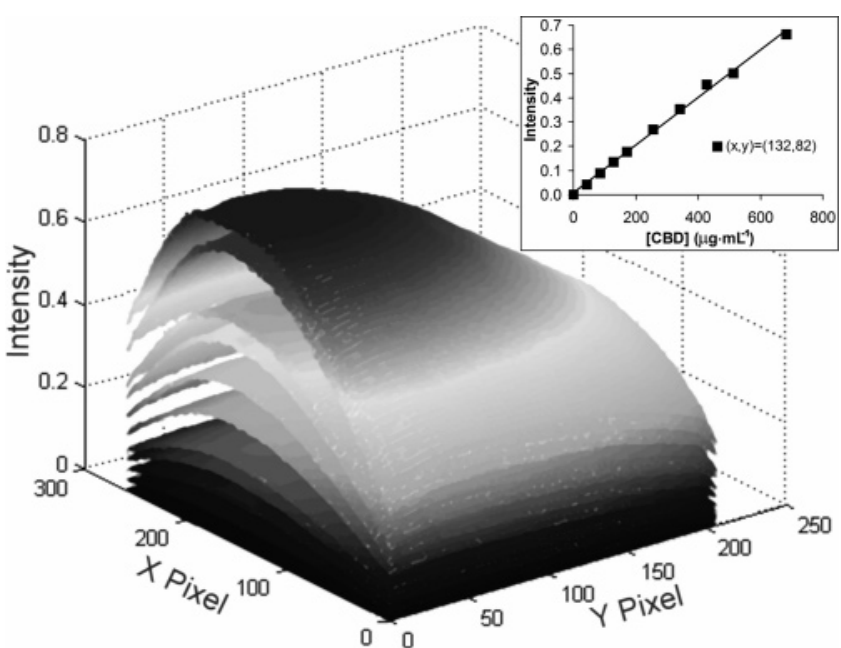

Figure 4. Calibration images obtained at capture time of $300 \mathrm{~ms}$; each surface represents a different CBD-FITC concentration. The inset shows, as an example, the CBD concentration versus pixel intensity, obtained approximately at the center of the images.
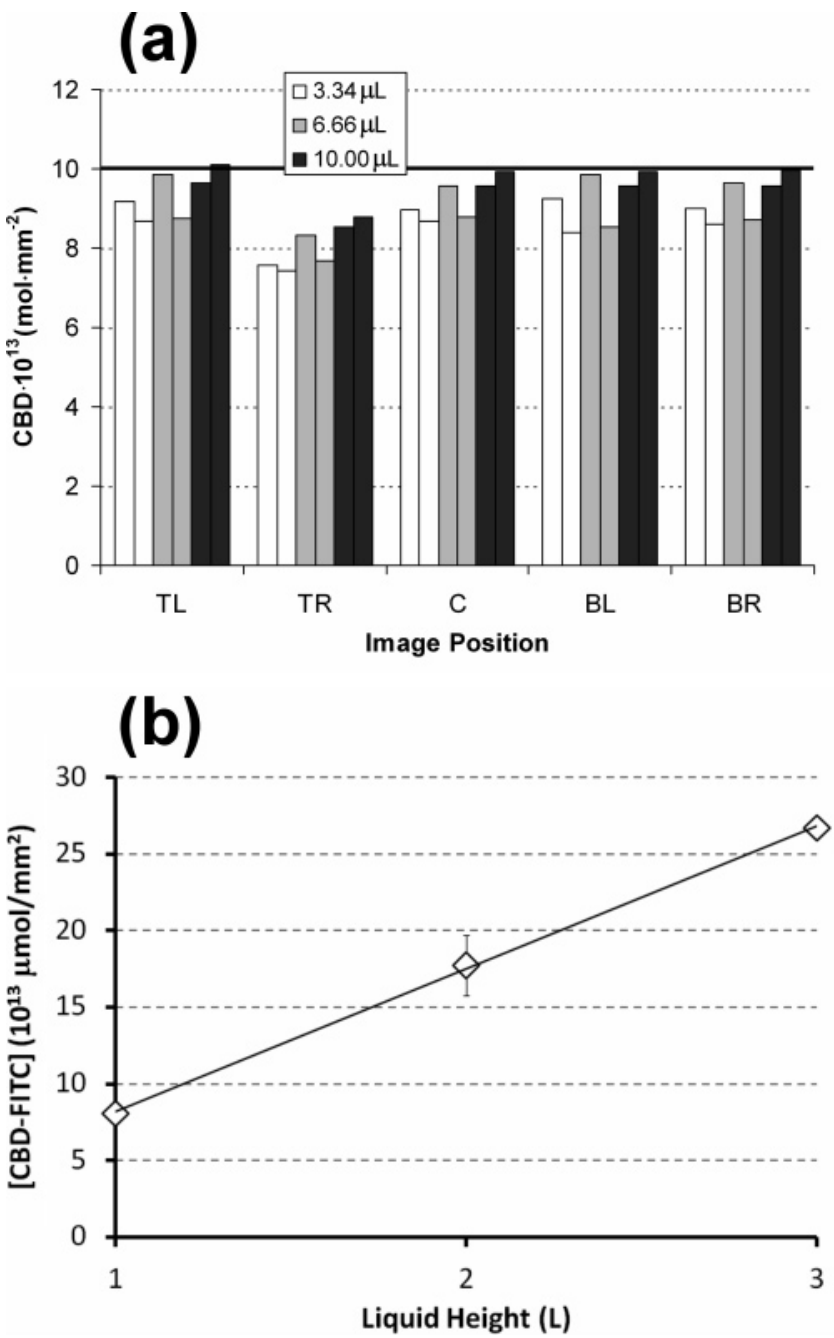

Figure 5. (a) Estimated CBD-FITC per unit area for three different protein solutions volumes. The labeling classes (TL, top left; TR, top right; $\mathrm{C}$, center; $\mathrm{BL}$, bottom left; $\mathrm{BR}$, bottom right) refer to average values obtained at different selected regions in the image. The black line corresponds to the expected value. Duplicate values are shown for each volume used. (b) CBD-FITC concentration, per unit area, calculated for three different heights of protein solution. These values are averages of several regions in the images; the linear regression has an $r^{2}$ of 0.999 .

ing of the CBD-FITC adsorbed to a cellulose surface was analyzed. In the case of adsorbed protein, the $k_{\mathrm{d}}$ determination implies special care. Image acquisition for different exposure times cannot be carried out on the same surface spot because of photobleaching accumulation. On the other hand, because the surface is heterogeneous, variations in the fluorescence intensity may occur if different spots of the surface are chosen (for analysis using different exposure times). To overcome these difficulties, the following method was adopted: two images were recorded (acquisition time of $400 \mathrm{~ms}$ ) $20 \mathrm{~s}$ apart, during which the film was continuously exposed to fluorescence light, as to photobleach the fluorophore. Considering that the acquisition time was much smaller than the total time, the intensity measured in the first image could be considered equivalent to the initial fluorescence $\left(I_{0}\right)$, and the second image to the fluorescence $(I)$ after $20 \mathrm{~s}(t)$. Equation 4 allows, using these values, the calculation of $k_{\mathrm{d}}$. The images used in this experiment are shown in Figure 6. The fluorescence intensities measured along with the calculated $k_{\mathrm{d}}$ are presented in Table 2. The average value of $k_{\mathrm{d}}$ corresponds to a low photobleaching rate: an exposure time of $1 \mathrm{~s}$ implies a decrease of $0.42 \%$ fluorescence 


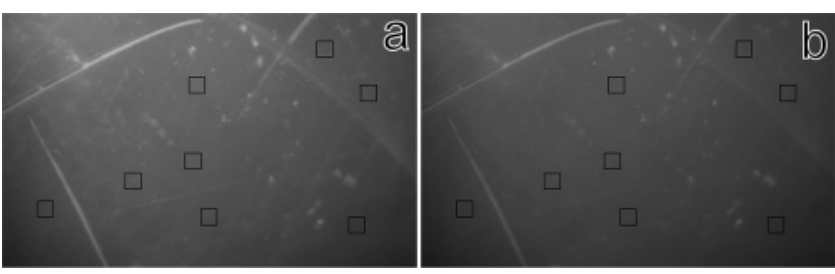

Figure 6. Cellulose film images obtained for $400 \mathrm{~ms}$ of exposure time at 0 (a) and $20 \mathrm{~s}$ (b) of exposure to the fluorescence light. The black squares indicate the positions where the measurements were carried out.

Table 2. Fluorescence Intensity at $0\left(I_{0}\right)$ and $20 \mathrm{~s}(I)$ of Continuous Exposure to Fluorescence Light and Calculated Photobleaching Constant Rate $\left(\boldsymbol{k}_{\mathrm{d}}\right)$

\begin{tabular}{ccc}
\hline $\mathrm{I}_{0}$ & $\mathrm{I}$ & $k_{\mathrm{d}}\left(\mathrm{s}^{-1}\right)$ \\
\hline 107.7 & 91.74 & 0.00804 \\
99.32 & 83.78 & 0.00850 \\
125.6 & 104.9 & 0.00904 \\
111.5 & 94.58 & 0.00822 \\
111.3 & 93.69 & 0.00863 \\
98.73 & 83.07 & 0.00864 \\
74.95 & 63.88 & 0.00799 \\
88.15 & 73.95 & 0.00878 \\
average & \multicolumn{2}{c}{$0.00848 \pm 0.00031$}
\end{tabular}

intensity $\left(I_{0} \times t\right)$. Therefore, in the course of fluorescence intensity measurements, the photobleaching of adsorbed CBDFITC may be neglected, under the experimental conditions of this work.

Figure 7 shows images of cellulose films with adsorbed CBD-FITC, where the black regions that are surrounded by pure white pixels correspond to saturated areas of intensity superior to the value of the highest CBD-FITC concentration used in the calibration. A CBD concentration expected to lead to saturation was used $\left(400 \mu \mathrm{g} \cdot \mathrm{mL}^{-1}\right.$, the equivalent to $20 \mathrm{mg}$ of CBD per gram of fibers). Figure 8 shows the adsorption isotherm for CBDs adsorbed on Whatman CF11. The gray circle represents the equilibrium position for $\mathrm{CBD}$ at the initial concentration of $400 \mu \mathrm{g} \cdot \mathrm{mL}^{-1}$. At this concentration, the CF11 fibers are saturated. Considering the much lower surface area of the material used in this work (a cellulose film instead of microparticles), the CBDs are expected to saturate the surface of the film. The cellulose films were produced by evaporation on a glass surface of cellulose acetate dissolved in acetone, which is likely to have scratches that may explain the highly fluorescent lines seen in the images (higher concentration of CBD-FITC adsorbed). These regions were therefore ignored in the concentration calculations, as shown in Figures 6 and 7. The average intensity values obtained for the selected regions in Figure 7 correspond to a concentration of CBD between 10.7 and $12.8 \times 10^{-13} \mathrm{~mol} \cdot \mathrm{mm}^{-2}$.

When observing a cellulose film (or fiber), the fluorescence detected may be produced by fluorescent molecules present on both sides of the film (fiber). For quantitative purposes, it is important to clarify whether the optical path through the fiber affects the intensity observed. An experiment was carried out (as described in Materials and Methods) to clarify this issue: a cellulose film with CBD-FITC adsorbed on only one of the sides was analyzed, exposing the surface with adsorbed CBDs directly to the digital camera in the microscope, or upside down. The intensity difference detected is below 5\%. Then, it can be considered that the total fluorescence has a similar contribution from the CBDs adsorbed on both sides of the cellulose film. Considering a CBD with $3.0 \times 1.8 \mathrm{~nm}(19)$, a monolayer of CBDs is expected to correspond approximately to $3.08 \times 10^{-13}$ $\mathrm{mol} \cdot \mathrm{mm}^{-2}$. It is then possible to calculate 1.7-2.1 layers of
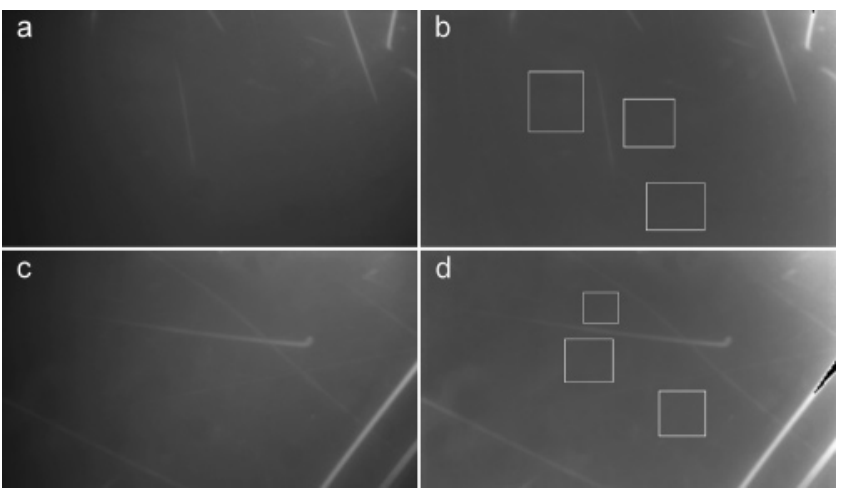

Figure 7. Images of cellulose films with adsorbed CBD-FITC: (a) and (c) correspond to gray scale images of the films, produced by the fluorescent light emitted by the CBD linked FITC; (b) and (d) correspond to the program treated images, where black color corresponds to $0 \mathrm{~mol} \cdot \mathrm{mm}^{-2}$ and white color to the maximum concentration used in the corresponding calibration procedure.

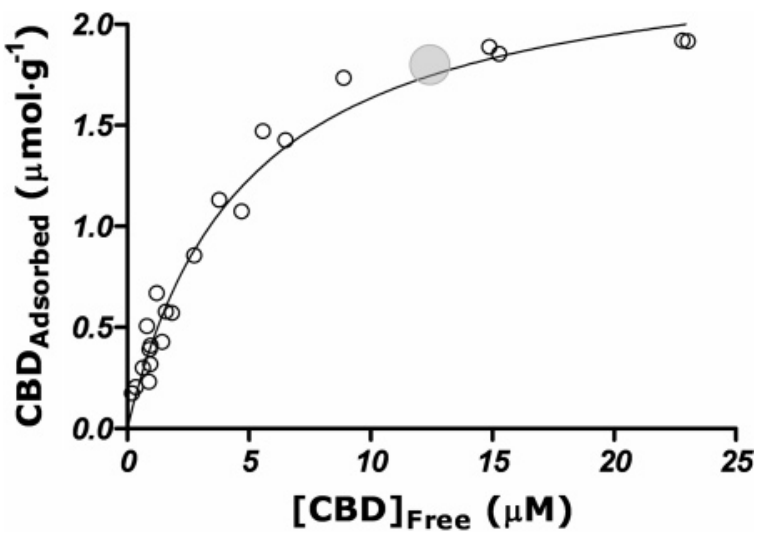

Figure 8. Adsorption isotherm of CBD on Whatman CF11 fibers, for $2 \mathrm{~h}$ contact. The line was obtained by nonlinear regression of the Langmuir isotherm, resulting in the constants: $\mathrm{CBD}_{\mathrm{Max}}=2.42 \pm 0.21$ $\mu \mathrm{mol} \cdot \mathrm{g}^{-1}$ and $k_{\mathrm{a}}=0.209 \pm 0.046 \mu \mathrm{M}^{-1}$. The gray circle corresponds to the equilibrium position of samples put in contact with an initial CBD concentration of $400 \mu \mathrm{g} \cdot \mathrm{mL}^{-1}$.

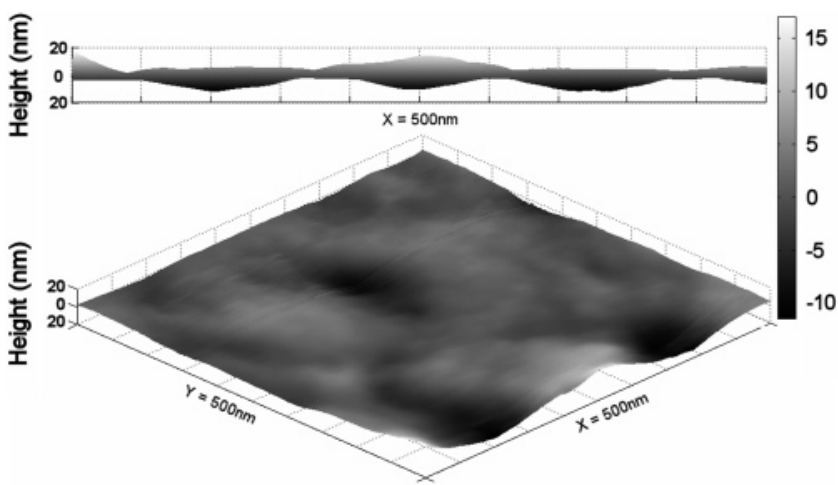

Figure 9. AFM surface image of cellulose film, obtained in the air The upper image corresponds to the $x x$ side view.

CBD in each side of the film. As seen in Figure 9, the surface of the cellulose acetate film has very low roughness, and this would increase only slightly the estimated surface concentration. A more plausible explanation, however, is advanced by Lee et al. (20), according to whom CBDs from $T$. reesei $\mathrm{CBHI}$ are able to penetrate cotton fibers. The film used in this work has low cristallinity (68\%) and may allow the penetration of surface diffusing CBDs. This would raise the adsorbed amount of CBD. Indeed, the presence of $\mathrm{CBD}$ multilayers in the surface of the 
film in unlikely. Rather, the CBDs are adsorbed in the external surface, but may also penetrate to other (internal) layers of the material.

\section{Conclusions}

A methodology was developed, based on the image analysis of adsorbed CBD-FITC conjugates, which allow the estimation of the protein surface concentration. This method is based on the production of a gray-scale image, obtained using the merging function $I=0.208 \times I_{\mathrm{R}}+0.695 \times I_{\mathrm{G}}+0.096 \times I_{\mathrm{B}}$. The calculated value of intensity is correlated with CBD-FITC concentration in a $x y$ plane. This correlation elicits the estimation of CBDs adsorbed in any kind of cellulosic material (particle, fiber, etc.). It has been shown that the photobleaching of adsorbed CBD-FITC is not relevant in the assay conditions. Preliminary results suggest that at saturation the CBDs adsorbed in a cellulose film accumulate in multiple layers. Other hypothesis to explain the high CBD concentration may be the protein penetration of the cellulose fibers. These issues will be studied in a future investigation.

\section{Acknowledgment}

Ricardo Pinto was supported by Fundação para a Ciência e a Tecnologia (FCT) grant SFRH/BD/6934/2001. The authors thank also the funding from FCT through the project POCTI/ AGRO/38253/2001.

\section{References and Notes}

(1) Srisodsuk, M.; Reinikainen, T.; Penttila, M.; Teeri, T. T. Role of the interdomain linker peptide of Trichoderma reesei cellobiohydrolase-I in its interaction with crystalline cellulose. J. Biol. Chem. 1993, 268, 20756-20761.

(2) Black, G. W.; Rixon, J. E.; Clarke, J. H.; Hazlewood, G. P.; Ferreira, L. M. A.; Bolam, D. N.; Gilbert, H. J. Cellulose binding domains and linker sequences potentiate the activity of hemicellulases against complex substrates. J. Biotechnol. 1997, 57, 59-69.

(3) Limón, M. C.; Margolles-Clark, E.; Benitez, T.; Penttila, M Addition of substrate-binding domains increases substrate-binding capacity and specific activity of a chitinase from Trichoderma harzianum. FEMS Microbiol. Lett. 2001, 198, 57-63.

(4) Srisodsuk, M. Mode of Action of Trichoderma reesei Cellobiohydrolase I on Crystalline Cellulose; VTT Publications: Helsinki, Finland, 1994; Vol. 188, pp 1-107.

(5) Mattinen, M. L.; Linder, M.; Teleman, A.; Annila, A. Interaction between cellohexaose and cellulose binding domains from Trichoderma reesei cellulases. FEBS Lett. 1997, 407, 291-296.

(6) Carrard, G.; Koivula, A.; Soderlund, H.; Beguin, P. Cellulosebinding domains promote hydrolysis of different sites on crystalline cellulose. Proc. Natl. Acad. Sci. U.S.A. 2000, 97, 10342-10347.

(7) Pala, H.; Lemos, M. A.; Mota, M.; Gama, F. M. Enzymatic upgrade of old paperboard containers. Enzyme Microb. Technol. 2001, 29, 274-279.
(8) Hildén, L., Daniel, G., Johansson, G. Use of a fuorescence labelled, carbohydrate-binding module from Phanerochaete chrysosporium Cel7D for studying wood cell wall ultrastructure. Biotechnol. Lett. 2003, 25, 553-558.

(9) Wierzba, A.; Reichl, U.; Turner, R. F. B.; Warren, R. A. J.; Kilburn, D. G. Adhesion of mammalian-cells to a recombinant attachment factor, $\mathrm{Cbd} / \mathrm{Rgd}$, analyzed by image-analysis. Biotechnol. Bioeng. 1995, 46, 185-193.

(10) Levy, I.; Shoseyov, O. Expression, refolding and indirect immobilization of horseradish peroxidase (HRP) to cellulose via a phage-selected peptide and cellulose-binding domain (CBD). J. Pept. Sci. 2001, 7, 50-57.

(11) Pinto, R.; Carvalho, J.; Mota, M.; Gama, M. Large-scale production of cellulose-binding domains. Adsorption studies using CBDFITC conjugates. Cellulose 2006, 13, 557-569.

(12) Kim, D. W.; Jang, Y. H.; Kim, C. S.; Lee, N. S. Effect of metal ions on the degradation and adsorption of two cellobiohydrolases on microcrystalline cellulose. Bull. Korean Chem. Soc. 2001, 22, $716-720$.

(13) Markham, J.; Conchello, J. A. Artefacts in restored images due to intensity loss in three-dimensional fluorescence microscopy. $J$. Microsc. (Oxford) 2001, 204, 93-98.

(14) Ghauharali, R. I.; Brakenhoff, G. J. Fluorescence photobleachingbased image standardization for fluorescence microscopy. J. Microsc. (Oxford) 2000, 198, 88-100.

(15) Song, L. L.; vanGijlswijk, R. P. M.; Young, I. T.; Tanke, H. J. Influence of fluorochrome labeling density on the photobleaching kinetics of fluorescein in microscopy. Cytometry 1997, 27, 213223.

(16) Zwier, J. M.; Van Rooij, G. J.; Hofstraat, J. W.; Brakenhoff, G. J. Image calibration in fluorescence microscopy. J. Microsc. (Oxford) 2004, 216, 15-24.

(17) Benson, D. M.; Bryan, J.; Plant, A. L.; Gotto, A. M.; Smith, L. C. Digital Imaging fluorescence microscopy-Spatial heterogeneity of photobleaching rate constants in individual cells. J. Cell Biol. 1985, 100, 1309-1323.

(18) Ghauharali, R. I.; Hofstraat, J. W.; Brakenhoff, G. J. Fluorescence photobleaching-based shading correction for fluorescence microscopy. J. Microsc. (Oxford) 1998, 192, 99-113.

(19) Kraulis, P. J.; Clore, G. M.; Nilges, M.; Jones, T. A.; Pettersson, G.; Knowles, J.; Gronenborn, A. M. Determination of the 3-dimensional solution structure of the C-terminal domain of cellobiohydrolase-I from Trichoderma reesei-A study using nuclear magneticresonance and hybrid distance geometry dynamical simulated annealing. Biochemistry 1989, 28, 7241-7257.

(20) Lee, I.; Evans, B. R.; Woodward, J. The mechanism of cellulase action on cotton fibers: evidence from atomic force microscopy. Ultramicroscopy 2000, 82, 213-221.

Received January 24, 2007. Accepted August 2, 2007.

BP070026V 